Investigation into the effect of feline hyperthyroidism on rectal temperature

\author{
Aisling McGinnity, Emma Roberts, Alix R \\ McBrearty \\ University of Glasgow, Glasgow, UK
}

Hyperthyroidism is reported to cause pyrexia of unknown origin. In one study, 19\% of hyperthyroid cats had an increased body temperature, however we rarely observe increased rectal temperature (RT) in our hyperthyroid cats. The aims of this study were to document the RT of hyperthyroid cats pre- and post-treatment and compare their median RT and the proportion of hyperthermic/pyrexic cats to a control population. A further aim was to investigate the correlation between $\mathrm{RT}$ and total $\mathrm{T} 4$.

Data from cats presented to a veterinary teaching hospital between 1/1/2010 and 1/10/2015 were reviewed. Pretreatment hyperthyroid cats were included if total T4 $>50 \mathrm{nmol} / \mathrm{l}$ and a RT measured within +/-6 days were recorded. Post-treatment cases were included if, following management, total T4 $<50 \mathrm{nmol} / \mathrm{L}$ and a RT was recorded within +/-6 days. A control group consisted of cats with the subsequent case number, a recorded RT and no record of hyperthyroidism. Kolmogirov-Smirnov tests of normality were performed and Pearson's correlation, Z-test and Mann-Whitney $U$ tests were performed when appropriate. $p<0.05$ was considered significant.

The median total T4 of 117 cats with uncontrolled hyperthyroidism was $185 \mathrm{nmol} / \mathrm{L}$ (range: $50.3-990 \mathrm{nmol} / \mathrm{L}$ ). Their median RT $38.2^{\circ} \mathrm{C}$ (range: $36.9-40.1^{\circ} \mathrm{C}$ ). Seven (5.98\%) were hyperthermic/pyrexic $\left(\mathrm{RT}>39.2^{\circ} \mathrm{C}\right)$. There was no correlation between RT and total T4 (Pearson's correlation coefficient $0.018, p=0.85$ ). Twenty six of these cats had an RT recorded when they were no longer hyperthyroid (total $\mathrm{T} 4<50 \mathrm{nmol} / \mathrm{L})$. The median RT at this time was $37.8^{\circ} \mathrm{C}$ (range: $36.7-38.9^{\circ} \mathrm{C}$ ) which was a median of $0.5^{\circ} \mathrm{C}$ (range: -1.3 to $+1.6^{\circ} \mathrm{C}$ ) lower than their RT pre-treatment $(p=0.003)$. The median temperature of the 70 cats in the control group was $38.4^{\circ} \mathrm{C}$ (range: $33.0-40.6^{\circ} \mathrm{C}$ ). This was not significantly different to the median RT of the hyperthyroid cats pre-treatment $(p=0.66)$. Seven $(10.00 \%)$ of the control cats were hyperthermic/pyrexic which was not significantly different to the proportion of hyperthermic/pyrexic cats in the pre-treatment hyperthyroid group.

Six percent of hyperthyroid cats had hyperthermia/pyrexia pre-treatment which was no different to the proportion in our control population. The hyperthyroid cat's pre-treatment RTs were not correlated with total T4. Following successful treatment, there was a significant reduction in RT. This suggests that high total T4 may increase the cat's RT but it rarely results in temperatures above the normal range and other causes of pyrexia/hyperthermia should be sought in these cats.

\section{The Diabetic Clinical Score (DCS): evaluation of a simple standardised quantification tool to allow rapid description of clinical signs in diabetic cats \\ Fernanda Venzon Varela ${ }^{1,3}$, Ruth Gostelow', Yaiza Forcada', David Church', Stijn Niessen ${ }^{1,2}$}

\author{
1 The Royal Veterinary College, London, UK \\ 2 Newcastle Medical School, Newcastle Upon Tyne, UK \\ 3 Universidade Federal do Rio Grande do Sul, Porto Alegre, Brazil
}

Glycaemic parameters, such as fructosamine, glycosylated haemoglobin and serial glucose measurements (SGMs), should always be interpreted in light of the clinical signs exhibited by diabetic pets. Sample artefacts, assay-issues, inter-day variability and stress can yield deceiving laboratory results. Likewise, the clinical history can prove unreliable due to inter-person variation, due to varying ways of asking or answering questions, interpreting non-standardised language, forgetting questions and lack of quantification. The aim of this study was to evaluate the Diabetic Clinical Score (DCS), newly designed in an attempt to overcome the latter limitations.

During regular history taking, cat owners were asked to choose the specific severity rating (ranging from none/normal, mild, moderate to severe) of specific diabetic clinical signs (polyuria and polydipsia, polyphagia, attitude/activity).
The extent of possible weight loss was established by weighing (none; mild: <5\%; moderate: 5-10\%; severe: >10\%). The severity was subsequently converted into a score ranging from 0 (none/ normal) to 3 (severe), yielding a summarised total score (DCS) for all signs combined ranging from 0 (no clinical signs) to 12 (maximum clinical signs). The DCS was correlated with the following objective parameters obtained at the same visit: fructosamine, average blood glucose during 24-hours SGMs (AvBG) and 24-hour water intake and urination frequency. Parameters were assessed for normality and appropriate descriptive and correlation statistics used.

In total, 222 consultations with owners of 48 diabetic cats were evaluated. Patients comprised $29 \mathrm{MN}$ and $19 \mathrm{FN}$ cats, with mean bodyweight $4.7 \pm 1.3 \mathrm{~kg}$ and mean age $11.4 \pm 3.1 \mathrm{yrs}$ at first presentation. Breeds included domestic shorthair $(n=34)$, domestic longhair $(n=7)$, Siamese $(n=2)$ and individual cats from other purebreeds $(n=5)$. Median DCS throughout the study was 1 (range: 0-11), mean fructosamine 407 $\pm 120 \mu \mathrm{mol} / \mathrm{l}$, mean AvBG $12.0 \pm 5.0 \mathrm{mmol} / \mathrm{l}$, median water intake $112 \mathrm{ml} / 24$ hours (range: 7-643) and median urination frequency 2 (range: $0-9$ ). The DCS showed significant correlations with fructosamine (Spearman's rho: 0.529, $p<0.001$ ), AvBG (Spearman's rho: 0.471, $p<0.001$ ), water intake (Spearman's rho: 0.453, $p<0.001)$ and urination frequency (Spearman's rho: 0.350, $\mathrm{p}<0.001$ ).

The DCS proved easy to comprehend by diabetic cat owners. The significant correlation between DCS and objective clinical parameters suggests it could represent a simple, more objective tool to describe, communicate and quantify the clinical signs encountered in diabetic cats in clinical practice and a research setting. Differences between the DCS and objective parameters should be further investigated. 